\title{
An Observational Cerebral Magnetic Resonance Imaging Study Following 7 Days at $4554 \mathrm{~m}$
}

\author{
Sven Kühn,, Darius Gerlach, ${ }^{2}$ Hans-Jürgen Noblé, ${ }^{1}$ Frank Weber, ${ }^{1}$ Jörn Rittweger, ${ }^{2}$ \\ Jens Jordan, ${ }^{2,3}$ and Ulrich Limper ${ }^{2,4}$
}

\begin{abstract}
Kühn, Sven, Darius Gerlach, Hans-Jürgen Noblé, Frank Weber, Jörn Rittweger, Jens Jordan, and Ulrich Limper. An observational cerebral magnetic resonance imaging study following 7 days at $4554 \mathrm{~m}$. High Alt Med Biol. 20:407-416, 2019.

Background: In human beings exposed to high altitude, cerebral magnetic resonance imaging (cMRI) revealed alterations ranging from subclinical cerebral edema formation to subtle brain abnormalities. Yet, brain structure after adaptation to high altitude and their recovery after return to lowlands have been rarely investigated. We, therefore, examined 10 healthy individuals by cMRI before, 12 hours after descent $(\mathrm{R}+12 \mathrm{~h})$, and again 3.5 months $(\mathrm{R}+3.5 \mathrm{~m})$ after a 7-day high altitude exposure at $4554 \mathrm{~m}$.

Results: After their 3-day lasting, stepwise ascent to $4554 \mathrm{~m}$, all subjects suffered acute mountain sickness with a mean Lake Louise score of 5.8 \pm 1.7 after the first night at that altitude. Acute mountain sickness completely resolved after 4 days at $4554 \mathrm{~m}$. While 12 hours after descent mean white and gray matter volumes were increased compared with before altitude exposure $(p=0.045$ and $p=0.002)$, these volumes were normalized on $\mathrm{R}+3.5 \mathrm{~m}$. Moreover, we observed significant focal volume alterations likely attributed to either vasogenic or cytotoxic edema formation. Two subjects presented new brain findings after altitude exposure. In one individual the number of preexisting white matter hyperintensities (WMHI) transiently increased, in the other individual a reversible splenial lesion syndrome (RESLES) emerged. Both findings had resolved 15 and 8 days after descent, respectively. None developed structural lesions like brain atrophy, cerebral infarcts, microbleeds, or highaltitude cerebral edema.

Discussion: Three days after complete recovery from acute mountain sickness and after return to low altitude, subclinical vasogenic and cytotoxic edema, RESLES and WMHI are present in high-altitude acclimatized individuals. However, these cerebral alterations are reversible within months at lowland.
\end{abstract}

Keywords: acclimatization; cytotoxic edema; RESLES; vasogenic edema; white matter lesion

\section{Introduction}

$\mathbf{E}$ ACH YEAR TENS OF THOUSANDS of individuals travel to high mountain ranges (Government of Nepal Ministry of Culture, Tourism \& Civil Aviation, Planning \& Evaluation Division, Research \& Statistical Section, 2018) exposing the brain to substantial hypobaric hypoxia. Indeed, ascent to high altitudes between 4000 and 5000 m moderately reduces brain oxygenation (Sanborn et al., 2015). Thereby, human brain physiology is significantly altered during and after acclima- tization to altitudes above $4000 \mathrm{~m}$ (Villien et al., 2013). Although acute mountain sickness and high-altitude cerebral edema can develop under these conditions, subclinical cerebral findings like marginal white matter volume increase and concomitant cerebrospinal fluid reduction may represent a normal response of the human brain to high altitude (Verges et al., 2016).

However, after exposure to extreme altitudes, findings like cerebral microbleeds can be considered as footprints in the brain of severe hypoxia and high-altitude cerebral edema

\footnotetext{
${ }^{1}$ German Air Force Center of Aerospace Medicine, Fürstenfeldbruck, Germany.

${ }^{2}$ German Aerospace Center (DLR), Institute of Aerospace Medicine, Cologne, Germany.

${ }^{3}$ Institute of Aerospace Medicine, University of Cologne, Cologne, Germany.

${ }^{4}$ Department of Anesthesiology and Intensive Care Medicine, Merheim Medical Center, Hospitals of Cologne, University of Witten/ Herdecke, Cologne, Germany.
} 
(Schommer et al., 2013; Fanou et al., 2017). Yet, an association between other cerebral findings like the reversible splenial lesion syndrome (RESLES) or white matter hyperintensities (WMHI) and high-altitude exposure are still a matter of debate (Garcia-Monco et al., 2011; Schommer et al., 2012).

Remarkably, cerebral magnetic resonance imaging (cMRI) data assessing the recovery of brain findings in individuals returning from high but not extreme altitudes are rare (Verges et al., 2016). Indeed, whether cerebral findings are fully reversible after recovery at lowland has not been investigated. Therefore, we tested whether an unacclimatized 9-day stay at high altitude produces reversible intracerebral volume changes and irreversible findings. We obtained cMRI to investigate global and focal brain volume alterations. We applied apparent diffusion-weighted characterization of the volume alterations, and fluid-attenuated inversion recovery sequences for characterization of cerebral findings. Furthermore, we used a susceptibility-weighted imaging (SWI) sequence to search for cerebral microbleeds. Hence, we obtained measurements before, 12 hours after descent after 7 days at $4554 \mathrm{~m}$, and again after 3.5 months to assess reversibility of high altitude-induced brain lesions.

\section{Methods}

Ten low-altitude native individuals ( 5 women, 5 men, age $41 \pm 15$ years, height $173 \pm 11 \mathrm{~cm}$, mean body mass index $23 \pm 3 \mathrm{~kg} / \mathrm{m}^{2}$ ) participated in the study. They underwent cMRI examinations before their active ascent, within 12 hours after descent, and again after 3.5 months of recovery at lowlands. They were healthy and on no regular medication, except for hormonal contraceptives in four women. All subjects denied preacclimatization defined as altitude exposure above $3000 \mathrm{~m}$ within the last 6 months before study inclusion and they stayed below an altitude of $1000 \mathrm{~m}$ un- til they had completed all recovery brain MRI scans of this study. All subjects resided below $500 \mathrm{~m}$ and had never experienced either acute mountain sickness or high-altitude pulmonary or cerebral edema. However, tolerance against acute mountain sickness was neither an inclusion nor exclusion criterion.

The study complied with the 2013 version of the Declaration of Helsinki and was approved by the ethics committee of the Medical Council Nordrhein, Duesseldorf, Germany, which handled the study under the reference number 2016225. All subjects provided written informed consent before inclusion into the study.

Participants were exposed to high altitude in the Capanna Regina Margherita mountain hut at $4554 \mathrm{~m}$ altitude in the Italian Monte Rosa massif (Fig. 1). Before altitude exposure, each participant underwent baseline (PRE) measurements at the MRI laboratory of the German Aerospace Center (DLR) in Cologne, Germany, at $77 \mathrm{~m}$ of altitude. On their 3-day sojourn to the Capanna Margherita, they spent one night in Alagna, Italy, at $1154 \mathrm{~m}$. The next day, they reached Passo dei Salati ( $2980 \mathrm{~m}$ ) by cable car and descended actively to the Orestes hut at $2600 \mathrm{~m}$ and stayed there overnight. The following day, participants actively climbed to the Capanna Gnifetti mountain hut at $3647 \mathrm{~m}$ and stayed there for another night. The next day, they reached the Capanna Margherita by climbing in rope teams (Fig. 1). They stayed six nights at the Capanna Margherita and descended actively from the hut to the Punta Indren station at $3275 \mathrm{~m}$ to take the cable car further down to Alagna. Subsequently, participants travelled by car to the MRI department of the Center of Aerospace Medicine of the German Air Force in Fuerstenfeldbruck, Germany, at $517 \mathrm{~m}$ of altitude. There we obtained the first follow-up cMRI scans within 12 hours after descent $(\mathrm{R}+12 \mathrm{~h})$ to search for changes in brain volumina and water diffusivity. We obtained late follow-up cMRI scans 3.5 months after descent $(\mathrm{R}+3.5 \mathrm{~m})$ at the DLR in Cologne to investigate for reversibility of cerebral findings.

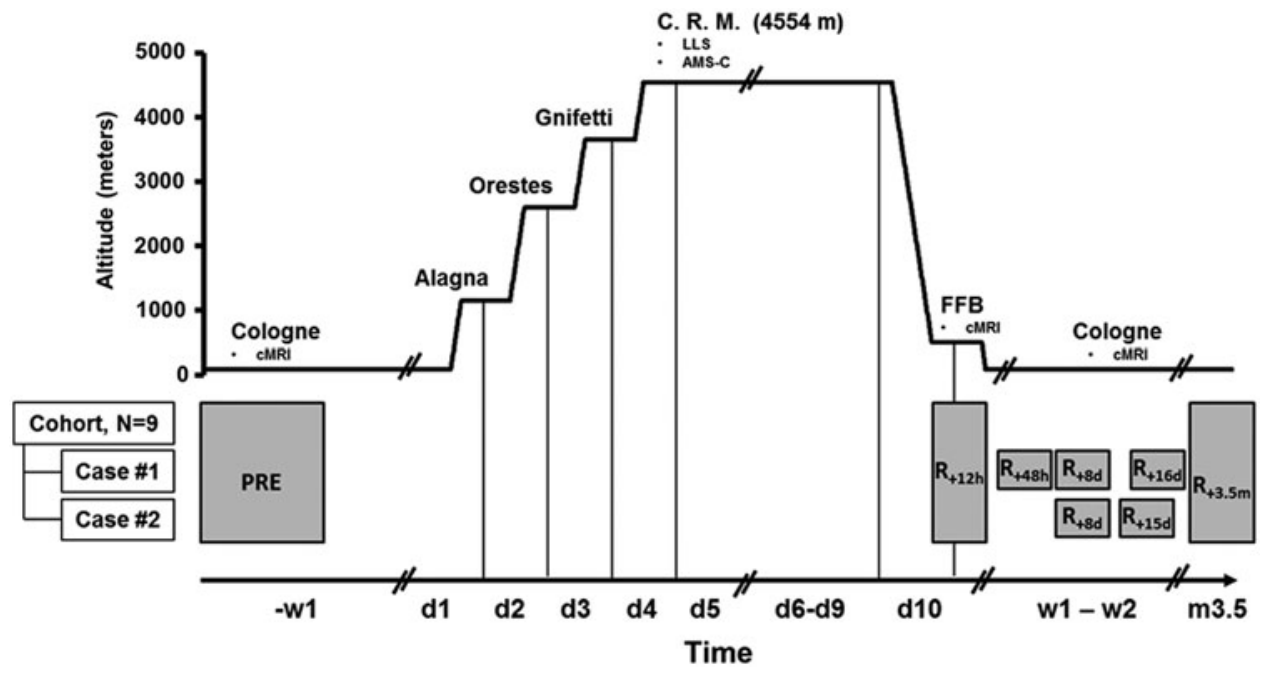

FIG. 1. Altitude profile and study setup. Baseline cMRI measurements (PRE) were performed 1 week before the ascent (-w1). Overnight stays at Alagna (d1, day 1), the Orestes hut (d2, day 2), and the Capanna Gnifetti (d3, day 3) preceded the high-altitude exposure (d4-d10) at the C.R.M. On the 10th study day (d10), the subjects descended and underwent their $\mathrm{R}+12 \mathrm{~h}$ brain MRI within 12 hours after leaving the C.R.M. at the MRI laboratory of the German Air Force in Fuerstenfeldbruck (FFB). Follow-up MRI measurement took place after 3.5 months after descent (R.3.5m). The two subjects with the brain findings (Case \#1 and Case \#2) underwent additional MRI exams after $48 \mathrm{~h}(\mathrm{R}+48 \mathrm{~h}), 8$ days $(\mathrm{R}+8 \mathrm{~d}), 15$ days $(\mathrm{R}+15 \mathrm{~d})$, or 16 days $(\mathrm{R}+16 \mathrm{~d})$ after descent. cMRI, cerebral magnetic resonance imaging; C.R.M., Capanna Regina Margherita. 
We estimated acute mountain sickness severity by the Lake Louise score (Roach et al., 1993) and the Acute Mountain Sickness-Cerebral (AMS-C) score and computed mean of the morning values (Sampson et al., 1983). We made the diagnosis acute mountain sickness at a Lake Louise score of three or more points, including at least one point from headache.

For cMRI imaging, we applied identical sequences at all time points. We obtained PRE and the $\mathrm{R}+3.5 \mathrm{~m}$ scans on a Biograph $\mathrm{mMR}^{\circledR}$ 3T scanner (with a 16-channel head coil; Siemens Healthcare, Erlangen, Germany) and $\mathrm{R}+12 \mathrm{~h}$ scans on a $3 \mathrm{~T}$ Prisma ${ }^{\circledR}$ scanner (20-channel head coil; Siemens Healthcare). The application of different MRI scanners for longitudinal brain MRI measurements is possible provided that quality assurance tests of the scanners are monitored (Deprez et al., 2018). A detailed description of the sequences contained in the cMRI protocol is given in the Supplementary Data.

We analyzed T1-weighted magnetization-prepared rapid acquisition of gradient echo (MPRAGE) images based on voxel morphology with the VBM8 toolbox of the SPM12 free software package. First, we segmented images into gray matter volumes, white matter volumes, and cerebrospinal fluid volumes. Thereafter, we added the three volumes to estimate total intracranial volumes. We excluded large intracranial vessels from tissue volume estimations. Then, images were diffeomorphic anatomical registration through exponentiated lie algebra normalized into the Montreal Neurological Institute (MNI) space (Ashburner, 2007) and smoothed with an 8-mm full width at half maximum Gaussian kernel.

Mean apparent diffusion coefficient values $\left(\mathrm{mm} / \mathrm{s}^{2}\right)$ were estimated on a Siemens syngo.plaza workstation system for the following brain regions of interest: bilaterally within the frontal white matter, the centrum semiovale, the lentiform nuclei, the cerebellar white matter, the thalami, the postcentral gyri, the paracentral lobules, and the genu and splenium of the corpus callosum. Susceptibility-weighted images were examined for pathological lesions by three independent experienced radiologists, who were blinded for measurement session, oxygen saturation values, and Lake Louise scores.

\section{Statistical analyses}

We constructed linear mixed-effects (LMEs) model fits by Restricted Maximum Likelihood estimations (REML) with time point as fixed and subject ID as random effect. The acute $(\mathrm{R}+12 \mathrm{~h})$ and the chronic $(\mathrm{R}+3.5 \mathrm{~m})$ effects of the highaltitude exposure on the Lake Louise and the AMS-C scores, the global volume changes and the changes of local water diffusivity of the intracranial compartments were analyzed. The taller and heavier participants had moderately higher total intracranial volumes compared with smaller participants of this study resulting in a bimodal distribution of the total intracranial volume (Fig. 2). Further information on this anecdotal finding can be found in the supplements (Supplementary Fig. S1). We included total intracranial volume as fixed factor into the LME model of the global intracranial volumes because it had proven as a significant factor for the analysis (Supplementary Table S1).

We performed simple linear regression analysis to estimate effects of intracranial volume alterations on acute mountain sickness severity measured by the cumulative AMS-C score of the first three mornings at $4554 \mathrm{~m}$. Statistical analyses were carried out in IBM SPSS Statistics. Data are given as mean and standard deviations (SDs). The level for statistical significance was set to $\alpha=0.05$.

To detect focal changes of the brain tissues, the smoothed segmented $\mathrm{T} 1$ volumetric images were further analyzed by parametric statistics that included general linear models and were based on the theory of Gaussian random fields (Ashburner and Friston, 2000). The Null hypothesis was tested that no intracranial volume changes would be detectable after high-altitude exposure. A repeated measure one-factorial analysis of variance with session as within-subject factor was applied in SPM. The first defined contrast of interest was a $\mathrm{t}$-contrast between the PRE and the $\mathrm{R}+12 \mathrm{~h}$ session, second, the t-contrast between PRE and $\mathrm{R}+3.5 \mathrm{~m}$, and third, the $\mathrm{t}$-contrast between $\mathrm{R}+12 \mathrm{~h}$ and $\mathrm{R}+3.5 \mathrm{~m}$. This analysis of multiple comparisons provided, after Bonferroni correction, a voxel map with significant local volume differences. Data are given as mean and SDs. The level for statistical significance was set to $\alpha=0.05$ after balancing for the family-wise error.

\section{Results}

At $4554 \mathrm{~m}$ altitude all subjects developed headache and acute mountain sickness reaching its maximum on the morning after the first night (mean Lake Louse score [LLS] $5.8 \pm 1.7$ and mean AMS-C $0.98 \pm 0.58$ ). One woman developed severe weakness and gastrointestinal symptoms and had to descend after the first night at $4554 \mathrm{~m}$. Her data were excluded from the statistical analysis. In the nine remaining participants, cumulative LLS and AMS-C scores over the first three mornings at $4554 \mathrm{~m}$ were $13 \pm 5$ and $2.15 \pm 1.5$, respectively.

Gray and white matter volumes were significantly increased at $\mathrm{R}+12 \mathrm{~h}$ compared with $\mathrm{PRE}(p=0.002$ and $p=0.045$ ) and had fully recovered at $\mathrm{R}+3.5 \mathrm{~m}$ (Table 1 ). A trend for a compensatory decrease in cerebrospinal fluid volume was observed at $\mathrm{R}+12 \mathrm{~h}(p=0.06)$. Regression analysis showed no significant relationship between global brain tissue volume changes at $\mathrm{R}+12 \mathrm{~h}$ compared with PRE and the cumulative AMS-C score of the first three mornings at $4554 \mathrm{~m}$ (Fig. 2).

We also observed local cerebral edema formation following high-altitude exposure (Fig. 3). On $\mathrm{R}+12 \mathrm{~h}$ compared with $R+3.5 \mathrm{~m}$, we detected significantly higher mean volumes in the left thalamus, the white matter next to the right putamen, the postcentral gyrus, and the right paracentral gyrus. On $\mathrm{R}+12 \mathrm{~h}$ compared with PRE, we detected significantly higher mean volumes in the left thalamus and the white matter next to the right putamen. Cluster-level, peak-level and MNI coordinates of these changes can be found in the Supplementary Data.

Shortly after high-altitude exposure, several brain tissue foci showed altered water diffusivity (Table 2). Mean apparent diffusion coefficients at $\mathrm{R}+12 \mathrm{~h}$ were significantly increased compared with PRE in the right lentiform nucleus and in the right and the left paracentral gyrus. Mean apparent diffusion coefficients at $\mathrm{R}+12 \mathrm{~h}$ were significantly increased compared with $\mathrm{R}+3.5 \mathrm{~m}$ in the right postcentral gyrus. An increase in mean apparent diffusion coefficient did not reach statistical significance in the left lentiform nucleus. In contrast the mean apparent diffusion coefficient of the splenium was lower at $\mathrm{R}+12 \mathrm{~h}$ compared with PRE and of the left thalamus at $\mathrm{R}+12 \mathrm{~h}$ compared with $\mathrm{R}+3.5 \mathrm{~m}$. A decrease in 

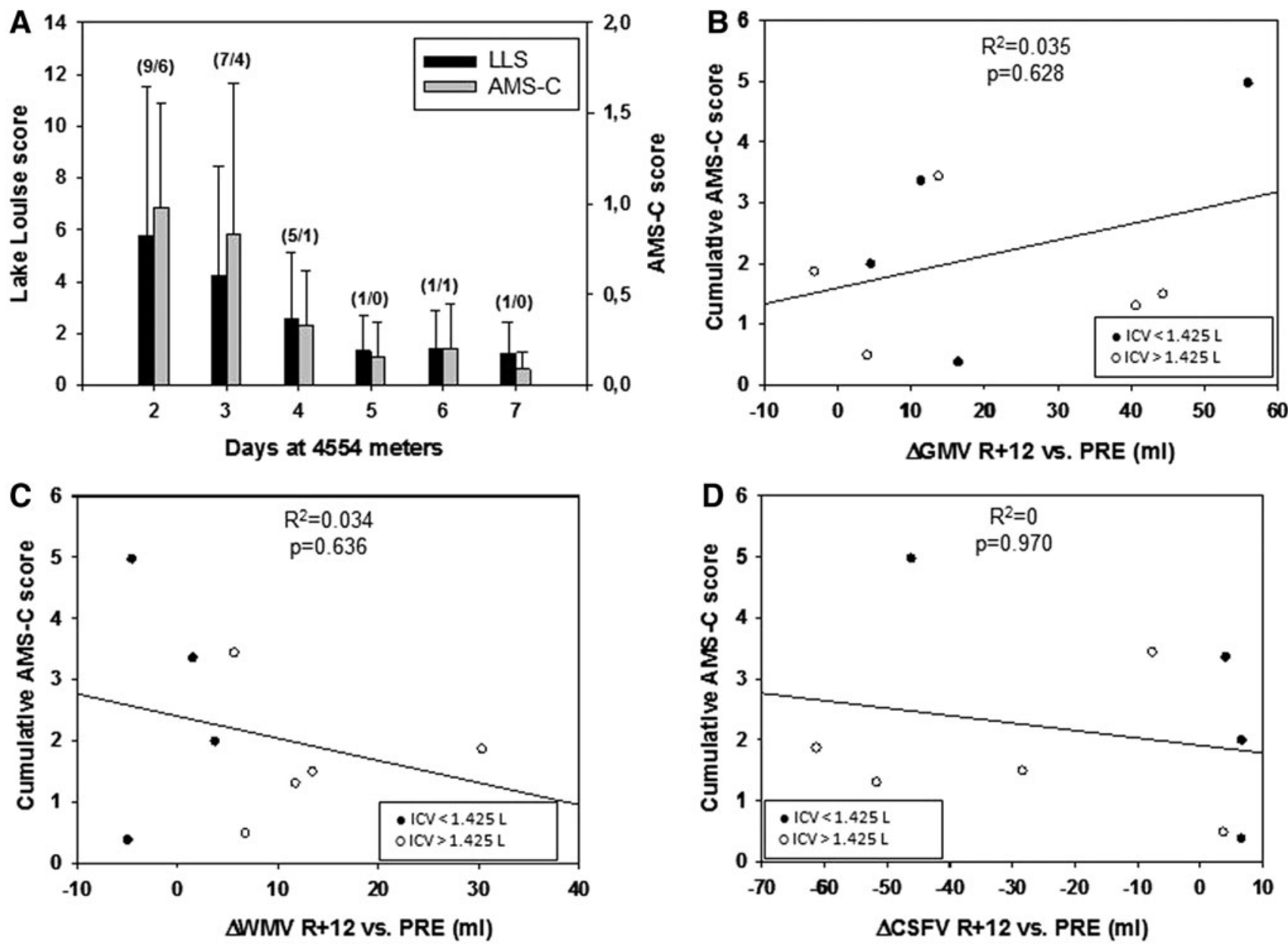

FIG. 2. Time course of acute mountain sickness severity at $4554 \mathrm{~m}$ and cerebral MRI alterations. (A) The mean LLS and the AMS-C score of the nine subjects, estimated during the morning hours, reach their maximums after the first night (day 2), and decrease thereafter continuously. Numbers in parentheses indicate the number of individuals each day reaching a LLS of $\geq 3$, including headache and an AMS-C score $\geq 0.7$, respectively. (B-D) Simple linear regressions were calculated to predict cumulative AMS-C scores over the first three mornings at $4554 \mathrm{~m}$ based on cerebral matter volume change on $\mathrm{R}+12 \mathrm{~h}$. No significant regression equations could be found. Closed circles indicate individuals with an ICV $<1.425 \mathrm{~L}$. Open circles indicate individuals with an ICV larger than 1.425 L. AMS-C, Acute Mountain Sickness-Cerebral; ICV, intracranial volume; LLS, Lake Louse score.

mean apparent diffusion coefficient did not reach statistical significance in the right thalamus. An increased and decreased mean apparent diffusion coefficient indicates vasogenic and cytotoxic edema formation, respectively.

Two participants developed abnormal brain findings at high altitude. One participant showed a small cytotoxic edema of the splenium, which disappeared within 16 days after descent (Fig. 4). He developed moderately acute mountain sickness, but no other symptoms typically associated with high-altitude cerebral edema or RESLES. During the first 48 hours at altitude the participant took ibuprofen repeatedly but no further drugs for the treatment of the symptoms of acute mountain sickness.

The second participant demonstrated an increase in the size and the number of pre-existing WMHI after his altitude stay. Based on their diffusivity, these hyperintensities were likely caused by vasogenic edema formation of the white matter at altitude or during descent. He experienced shortlasting, moderately acute mountain sickness without symptoms typically associated with high-altitude cerebral edema.

Table 1. Volumetric Changes in Intracranial Compartments

\begin{tabular}{|c|c|c|c|c|c|}
\hline Compartment & $P R E(m L)$ & $R+12 h(m L)$ & $\Delta R+12 h-P R E(\%)$ & $R+3.5 m(m L)$ & $\mathrm{p}$ \\
\hline WMV & $446 \pm 45$ & $453 \pm 53$ & $3.1 \pm 3.2$ & $447 \pm 48$ & 0.045 \\
\hline GMV & $685 \pm 31$ & $706 \pm 34$ & $1.5 \pm 2.3$ & $683 \pm 38$ & 0.002 \\
\hline CSFV & $309 \pm 75$ & $290 \pm 61$ & $-5.3 \pm 8.1$ & $308 \pm 81$ & 0.06 \\
\hline
\end{tabular}

The mean volumes \pm SD of the nine subjects are shown. Baseline, PRE, 12 hours after the return from 7 days at $4554 \mathrm{~m}(\mathrm{R}+12 \mathrm{~h}), 3.5$ months after the return from 7 days at $4554 \mathrm{~m}(\mathrm{R}+3.5 \mathrm{~m}), \Delta \mathrm{R}+12 \mathrm{~h}-\mathrm{PRE}$, percent volume changes at $\mathrm{R}+12 \mathrm{~h}$ compared with PRE.

CSFV, cerebrospinal fluid volume; GMV, gray matter volume; SD, standard deviation; WMV, white matter volume. 

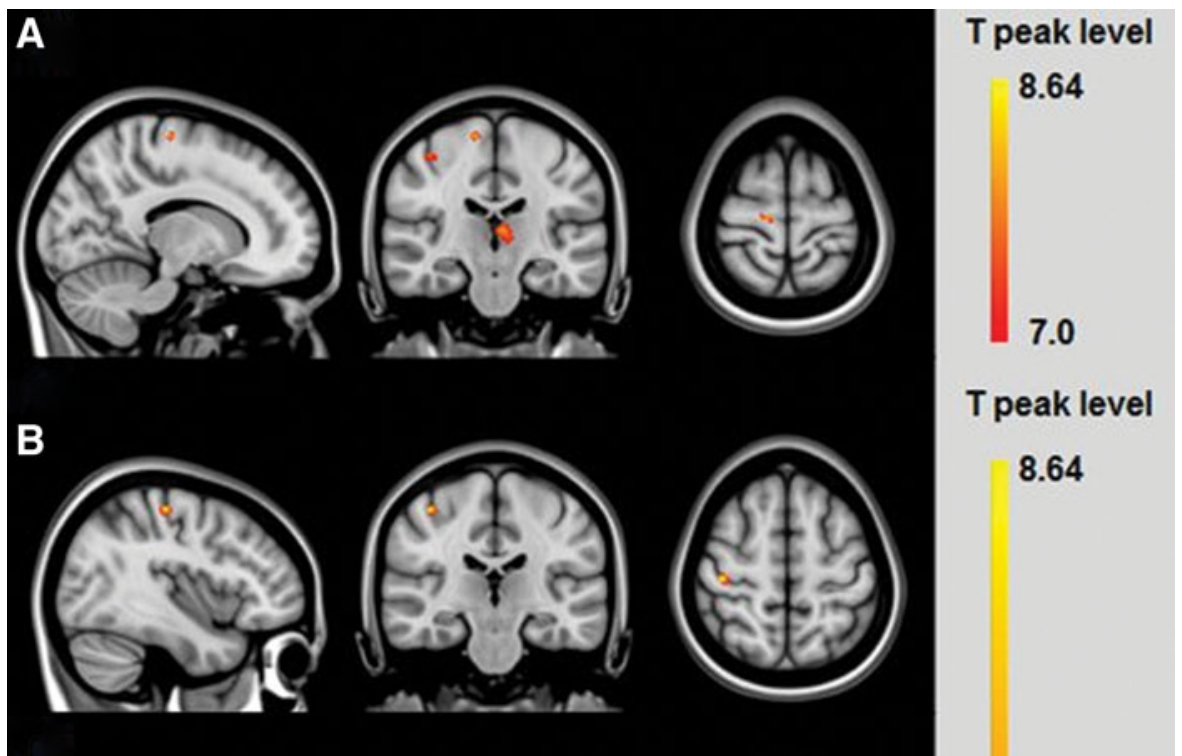

T peak level
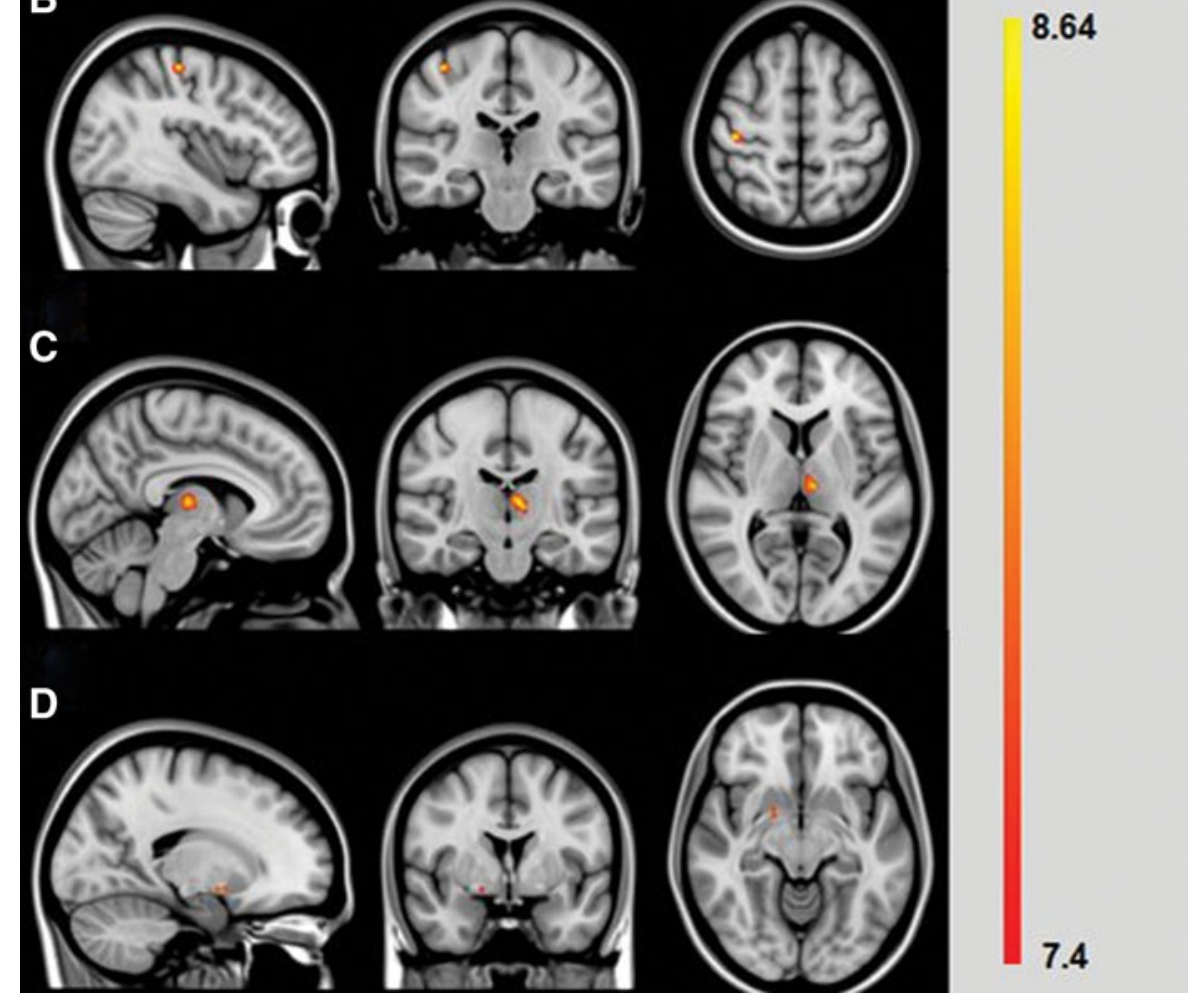

FIG. 3. Changes of focal cerebral tissue volumes during recovery from 7 days at $4554 \mathrm{~m}$. Focal cerebral tissue volumes are compared between 12 hours after descent $(\mathrm{R}+12 \mathrm{~h})$ and 3.5 months after descent $(\mathrm{R}+3.5 \mathrm{~m})$. Significant focal cerebral tissue volume changes $(p<0.05$, Bonferroni corrected) are found in: (A) paracentral gyrus region of the right brain; (B) postcentral gyrus region of the right brain; (C) thalamic area of the left brain; and (D) area of the putamen of the right brain. The results are overlaid on the Montreal Neurological Institute standard space brain template.

The participant did not take drugs for the treatment of acute mountain sickness symptoms. These findings were bilaterally located in the frontoparietal and dorsal deep white matter and showed a subcortical and periventricular distribution pattern (Fig. 5). The WMHI had fully vanished 15 days after descent.

None of the remaining participants developed abnormal brain findings at altitude. In particular, we detected no microbleedings by SWI.

\section{Discussion}

The important finding of our study is that 7 days at $4554 \mathrm{~m}$ elicit significant changes in mean global and focal volumes and water diffusivity of the human brain that outlast altitude exposure by at least 12 hours and acute mountain sickness symptoms by at least 3 days. While all participants developed acute mountain sickness, none progressed to high-altitude cerebral edema. However, a reversible splenial lesion in one and WMHI in another participant regressed within 2 weeks after descent. After 3.5 months, all altitude-associated brain alterations had fully vanished.

Significantly increased water diffusivity together with increased volumes of right-sided post- and paracentral gyrus and left thalamus suggest subclinical brain edema formation (Lawley et al., 2016). In previous studies, mechanisms mediating such brain volume shifts remained a matter of debate (Kallenberg et al., 2007; Wilson et al., 2011; Lawley et al., 2014; Verges et al., 2016; Sagoo et al., 2017). Another cMRI study reported significant increases in white matter volumes in individuals within the first 6 hours after return from 6 days at $4350 \mathrm{~m}$ altitude. Altered diffusion values indicated vasogenic edema formation, which was attributed to increased blood flow (Verges et al., 2016). The mechanisms mediating gray and white matter edema formation have also been investigated in studies simulating altitude by normobaric hypoxia (Morocz et al., 2001; Kallenberg et al., 2007; 
Table 2. Magnetic Resonance Imaging Apparent Diffusion Coefficient Dynamics in Cerebral Locations of InTERest

\begin{tabular}{|c|c|c|c|c|}
\hline Location of interest & $\operatorname{PRE}\left(\mathrm{mm} / \mathrm{s}^{2}\right)$ & $R+12 h\left(m m / s^{2}\right)$ & $R+3.5 m\left(\mathrm{~mm} / \mathrm{s}^{2}\right)$ & $\mathrm{p}$ \\
\hline Right frontal white matter $(=)$ & $768 \pm 22$ & $762 \pm 33$ & $770 \pm 27$ & 0.658 \\
\hline Left frontal white matter $(=)$ & $756 \pm 34$ & $760 \pm 38$ & $756 \pm 39$ & 0.856 \\
\hline Right centrum semiovale $(=)$ & $719 \pm 15$ & $714 \pm 41$ & $709 \pm 28$ & 0.770 \\
\hline Left centrum semiovale $(=)$ & $747 \pm 27$ & $747 \pm 27$ & $760 \pm 23$ & 0.325 \\
\hline Right lentiform nucleus $(\uparrow)$ & $677 \pm 39$ & $701^{a} \pm 26$ & $689 \pm 19$ & 0.043 \\
\hline Left lentiform nucleus $(\uparrow)$ & $688 \pm 26$ & $715 \pm 30$ & $693 \pm 36$ & 0.083 \\
\hline Right cerebellar hemisphere (=) & $639 \pm 32$ & $640 \pm 15$ & $632 \pm 21$ & 0.754 \\
\hline Left cerebellar hemisphere $(=)$ & $648 \pm 30$ & $663 \pm 18$ & $643 \pm 25$ & 0.116 \\
\hline Genu of corpus callosum $(=)$ & $793 \pm 60$ & $773 \pm 63$ & $804 \pm 53$ & 0.151 \\
\hline Splenium of corpus callosum $(\downarrow)$ & $769 \pm 50$ & $725^{\mathrm{a}} \pm 72$ & $755 \pm 58$ & 0.045 \\
\hline Right postcentral gyrus $(\uparrow)$ & $739 \pm 54$ & $783 \pm 64$ & $735^{a} \pm 32$ & 0.027 \\
\hline Left postcentral gyrus $(=)$ & $709 \pm 86$ & $735 \pm 71$ & $722 \pm 82$ & 0.219 \\
\hline Right paracentral gyrus $(\uparrow)$ & $710 \pm 48$ & $751^{b} \pm 41$ & $726^{\mathrm{a}} \pm 35$ & 0.001 \\
\hline Left paracentral gyrus $(\uparrow)$ & $723 \pm 37$ & $\mathbf{7 8 3}^{\mathrm{c}} \pm 37$ & $742 \pm 40$ & 0.005 \\
\hline Right thalamus $(\downarrow)$ & $732 \pm 20$ & $712 \pm 19$ & $720 \pm 25$ & 0.064 \\
\hline Left thalamus $(\downarrow)$ & $739 \pm 39$ & $731 \pm 29$ & $760^{\mathrm{a}} \pm 23$ & 0.041 \\
\hline
\end{tabular}

The mean $\mathrm{ADC} \pm \mathrm{SD}$ of brain regions of interest of the nine subjects are shown. Directions of changes in ADC values are indicated as follows: $=$, no change, $\uparrow$, increase, $\downarrow$, decrease. Bold $R+12 \mathrm{~h}$ values indicate a significant difference between the measurements at 12 hours after descent $(\mathrm{R}+12 \mathrm{~h})$ and baseline (PRE). Bold $\mathrm{R}+3.5 \mathrm{~m}$ values indicate a significant difference between the measurements at 3.5 months after descent $(\mathrm{R}+3.5 \mathrm{~m})$ and at 12 hours after descent $(\mathrm{R}+12 \mathrm{~h})$.

${ }^{\mathrm{a}} p<0.05,{ }^{\mathrm{b}} p<0.001,{ }^{\mathrm{c}} p<0.01, p$-values of the linear mixed-effects model fits with time point as fixed effect and balanced for the familywise error are given in the last column.

ADC, apparent diffusion coefficient.

Lawley et al., 2014; Sagoo et al., 2017). An increase in gray matter volume after 10 hours of simulated altitude was attributed to changes in cerebral venous blood volume (Lawley et al., 2014). Others observed significant increases in total brain volume after 16 hours of simulated altitude and implicated water accumulation in brain parenchyma (Kallenberg et al., 2007). In a 22 hours altitude simulation, cMRI revealed gray and white matter swelling. The authors suggested that hypoxia-driven increases in arterial cerebral blood flow coupled with impaired venous drainage ultimately increased intracranial hydrostatic pressure (Sagoo et al., 2017).

Our results show that swelling of the brain tissues persists even after 9 days of acclimatization at a high altitude. The prolonged duration of these abnormalities cannot be simply explained by transient changes in hydrostatic pressure conditions at high altitude. Our result of prolonged brain swelling after 9 days at high altitude and after acute mountain sickness symptoms had completely abated are supported by two recent publications. In the first study, white matter vasogenic edema continued to increase in the first week in patients suffering high-altitude cerebral edema even though patients were clinically improving (Hackett et al., 2019). The second study, a hypobaric chamber study, investigated a challenging 24-minute single hypobaric exposure at 25,000 feet, including 2-4 minutes with oxygen desaturation to $65 \%-75 \%$. The authors observed augmented white matter cerebral blood flow for 72 hours after exposure (McGuire et al., 2019).

Focal brain volume changes following high-altitude exposure (Zhang et al., 2013; Fan et al., 2016) have been rarely reported. In our study, edema-associated volumetric fluctuations were neither symmetrically nor otherwise evenly distributed. Four areas - the right postcentral gyrus, the right paracentral gyrus, the left thalamus, and a small area close to the putamen-showed significant volume increases. However, no participant showed relevant neurological impairments at altitude. Based on significant water diffusivity changes, edema formation is the likely underlying mechanism. Hypoxia-driven localized hyperperfusion might explain these focal volume increases. In healthy individuals, short-term normobaric hypoxia exposure elicited regional hyperperfusion determined by positron emission tomography in several brain regions, including hypothalamus, thalamus, and cerebellum. (Buck et al., 1998). Moreover, single-photon emission computed tomography revealed a side difference in regional cerebral blood flow responses in the right temporal lobe, anterior cingulate cortex, sensorimotor cortex, prefrontal cortex, and basal ganglia (Pagani et al., 2000, 2011). Possibly, the acute mountain sickness susceptible brain is characterized by impaired vascular autoregulation and, thus, more vulnerable to hyperperfusion-induced vasogenic edema (Swenson and Bärtsch, 2014). However, whether or not acute mountain sickness is causally related to vasogenic edema is still under debate. In our study, acute mountain sickness severity and brain volume changes were not directly related to each other. We cannot exclude that brain volumes, focal edema formation, and tissue water diffusivity changed after acute mountain sickness had resolved (Hunt et al., 2013). In any event, our study confirms that altitudeassociated vasogenic edema is unevenly distributed over different brain regions.

New brain lesions in two of our participants are a matter of concern. In the case with new WMHI at altitude, the lesions were exclusively located in regions of preexisting microangiopathic damage and were not related to more severe acute mountain sickness. The observation points to the importance of vascular barrier integrity in protecting the brain from edema formation. Latest research identified both edema and demyelination, possibly caused by breakdown of the blood-brain barrier and inflammation, as the major pathological substrates of WMHI (Iordanishvili et al., 2019).

The WMHI in our case were reversible. Several casecontrol studies revealed a high burden of WMHI in 


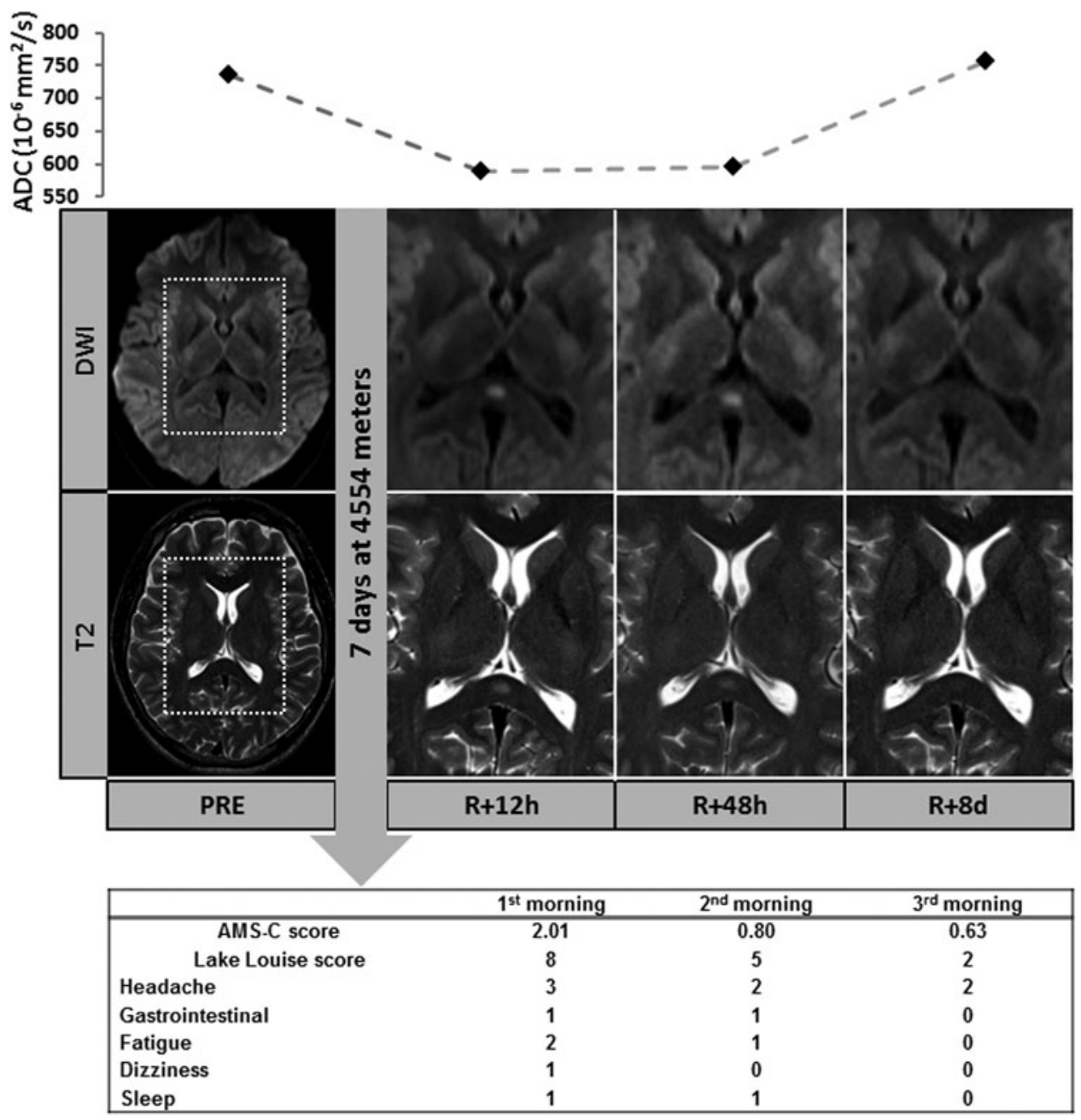

FIG. 4. Participant with reversible splenial cytotoxic edema (RESLES). At sea level (PRE), no RESLES is visible in the T2- and diffusion-weighted brain MRI sequences in the 30-year-old healthy, male subject. At altitude the participant presented acute mountain sickness during the first 48 hours only (Lake-Louise and AMS-C scores of 8 and 5 and of 2.0 and 0.8 after 24 and 48 hours, respectively). $12(\mathrm{R}+12 \mathrm{~h})$, and $48(\mathrm{R}+48 \mathrm{~h})$ hours after descend RESLES emerged and accompanied by a restricted diffusion and decreased ADC. RESLES occurs centrally and symmetrically, demonstrating a cytotoxic edema formation. Eight days later $(\mathrm{R}+8 \mathrm{~d})$, the splenium is recovered. This case suggests that RESLES may occur in acute mountain sickness without any other clinical symptoms. ADC, apparent diffusion coefficient; RESLES, reversible splenial lesion syndrome.

professional climbers (Garrido et al., 1993; Fayed et al., 2006; Zhang et al., 2012). Yet, lack of regular repeat imaging made it difficult differentiating reversible and permanent altitude-induced white matter lesions. A single prospective longitudinal study showed new white matter lesions in climbers $\sim 30$ days after high-altitude exposure (Garrido et al., 1995). The authors suggested that these lesions were irreversible, thus, reflecting scarred gliotic changes. While white matter lesion burden increases over time (de Leeuw and Nichols, 2017) and is associated with arterial hypertension, smoking, and diabetes (Wardlaw et al., 2015), white matter lesions secondary to acute vascular injury may be reversible (Wardlaw et al., 2017). Repeat imaging $\sim 2$ weeks after altitude exposure confirmed the transient nature of the white matter lesions in one of our subjects. We speculate that the phenomenon is explained by reduction of local edema after return from altitude.
In the second case, we observed reversible cytotoxic splenium edema, the so-called RESLES, after high-altitude exposure. This syndrome is associated with several pathologies (Garcia-Monco et al., 2011; Kühn et al., 2016), which is likely explained by predilection of the splenium for edema formation. The exact etiology has not yet been clarified. However, the corpus callosum appears to be particularly prone to hypoxia (Yamauchi et al., 1995). Both, vasogenic and cytotoxic edema have been implicated in severe high-altitude cerebral edema (Hackett et al., 1998, 2019). Our results are in line with others who also observed significant diffusion restrictions in the splenium of subjects suffering acute mountain sickness (Kallenberg et al., 2007). While only one of our subjects with acute mountain sickness showed a visible brain lesion, mean corpus callosum water diffusivity had significantly decreased in the whole group. Patients with severe acute mountain sickness or high-altitude cerebral edema were 


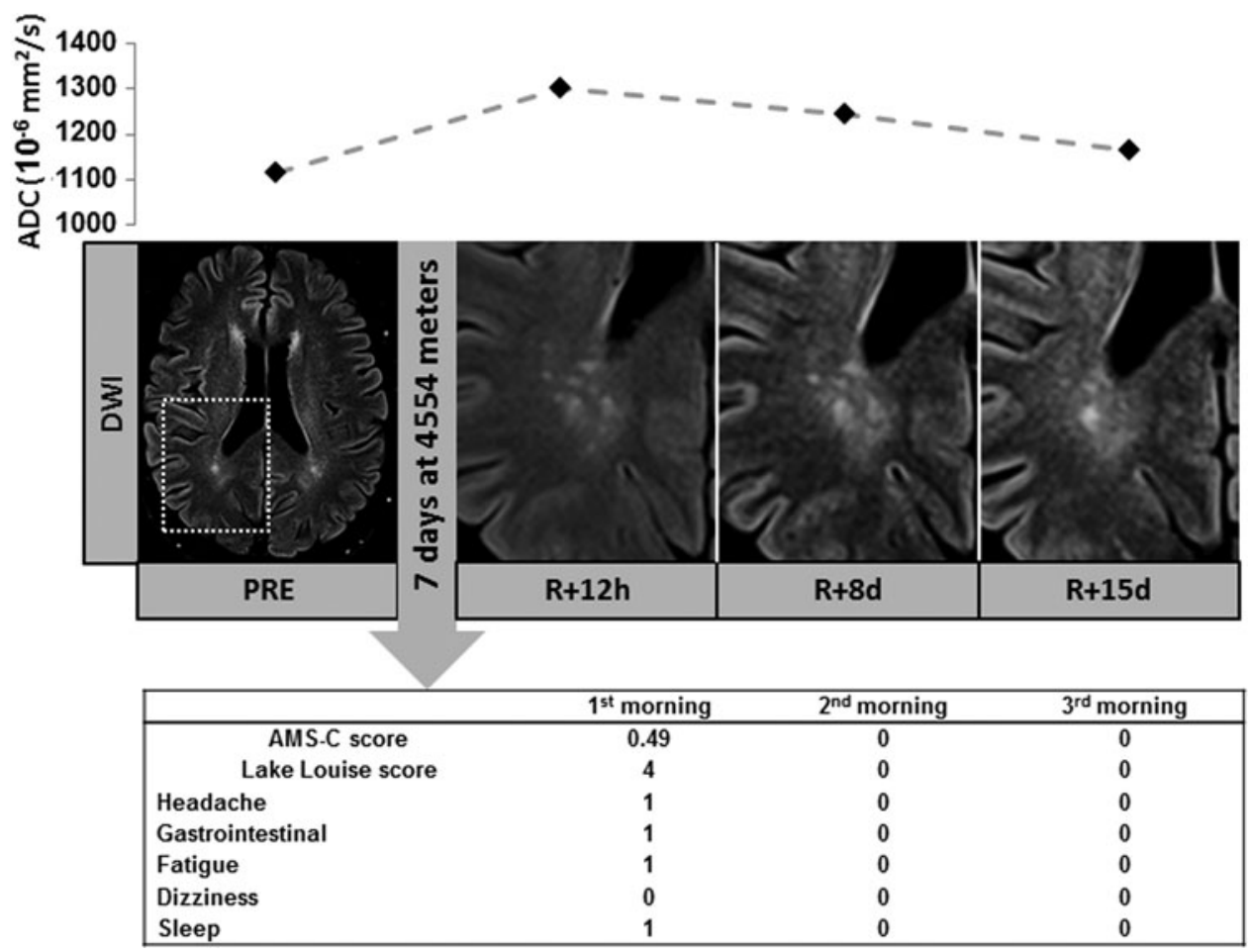

FIG. 5. Participant with reversible white matter lesions at $4554 \mathrm{~m}$ altitude. At sea level (PRE) a moderate number of white matter hyperintensities is visible in the FLAIR sequence in the 64-year-old healthy male subject. Image windows from the brain tissue dorsal of the posterior horn of the right ventricle show that $12(\mathrm{R}+12 \mathrm{~h})$ and $48(\mathrm{R}+48 \mathrm{~h})$ hours after descend, the white matter hyperintensities are emerged and accompanied by an increased ADC demonstrating vasogenic edema formation. Fifteen days later $(R+15 d)$ the emerged white matter hyperintensities had vanished. At altitude, the participant presented acute mountain sickness during the first 24 hours only (Lake-Louise-Score of 4). FLAIR, fluid-attenuated inversion recovery.

affected in published cases of RESLES after a high-altitude stay (Hackett et al., 1998; Bin and Lee, 2011). An association between lowered water diffusivity and acute mountain sickness severity was also described (Kallenberg et al., 2007; Schoonman et al., 2008; Hunt et al., 2013; Lawley et al., 2014; Sagoo et al., 2017). However, the exact time point of the development of RESLES in our subject is not identifiable. In addition to the possibility that the lesion has occurred at altitude, their emergence during descent cannot be excluded.

Our findings demonstrate that brain findings can occur at altitudes below $5000 \mathrm{~m}$. Yet, both cases illustrate that cytotoxic as well as vasogenic edema formation may occur under identical high-altitude climbing conditions. Yet, we did not observe cerebral microhemorrhages suggesting that the pathology is not very common in individuals with acute mountain sickness at $4554 \mathrm{~m}$.

The main limitation of our study is the small sample size, which precluded a detailed analysis of confounding factors affecting acute mountain sickness or brain structure. Indeed, high-altitude mountaineering elicits complex physiological changes especially in individuals developing acute mountain sickness. Physically challenged mountaineers are particularly vulnerable for acute mountain sickness possibly because physical activity level affects cerebral volume regulation (Mairer et al., 2012; Rupp et al., 2014). Gradual reoxygenation and recompression during transfer to the $\mathrm{R}+12 \mathrm{~h}$ measurements might have influenced brain physiology through increased oxygen stress and the formation of reactive oxygen species (Hoffmann et al., 2016). A recent report (Hackett et al., 2019) points into the same direction and raises the possibility of reoxygenation playing a role, since vasogenic and cytotoxic edema in some patients with high-altitude cerebral edema became worse over the first week. Other unknown effects during the descent may have also confounded our data. However, we transferred participants as fast as possible in this study setting. Finally, we may have overlooked mild-to-moderate neurocognitive dysfunction because we did not perform neurocognitive function testing. However, daily medical examinations did not reveal obvious neurological signs or symptoms.

Despite these issues, our study confirms that high-altitude mountaineering leads to reversible volumetric and circumscribed cerebral findings in individuals that are clinically unaffected by cerebral edema. Both, vasogenic and cytotoxic mechanisms seem to contribute to these cerebral alterations. Larger studies are needed to elucidate individual risk factors and to relate brain findings after altitude exposure to clinical symptoms.

\section{Acknowledgments}

The authors would like to thank the subjects, whose participation made this study possible. Furthermore, the authors gratefully acknowledge the technical support of Wolfram Sies, Joerg Holtschneider, Sina Boehne, Eva Krakor, and the administrative support of Christine Becker to this study. The authors are grateful to Andrea Enzio for mountain guiding. The authors express their appreciation to the teams operating 
Capanna Gnifetti and Capanna Margherita. The author would like to thank Dr. Stephen McGuire for his constructive criticism of the article.

\section{Author Disclosure Statement}

No competing financial interests exist.

\section{Funding Information}

This work was supported by the programmatic funding of the German Aerospace Center (DLR) and by a grant from the German Society for Mountain and Expedition Medicine (BExMed).

\section{Supplementary Material}

Supplementary Data

Supplementary Table S1

Supplementary Figure S1

\section{References}

Ashburner J. (2007). A fast diffeomorphic image registration algorithm. Neuroimage 38:95-113.

Ashburner J, and Friston KJ. (2000). Voxel-based morphometry-The methods. Neuroimage 11:805-821.

Bin CH, and Lee S-J. (2011). Teaching NeuroImages: Reversible splenial cytotoxic edema in acute mountain sickness. Neurology 77:e94.

Buck A, Schirlo C, Jasinsky V, Weber B, Burger C, von Schulthess GK, Koller EA, and Pavlicek V. (1998). Changes of cerebral blood flow during short-term exposure to normobaric hypoxia. J Cereb Blood Flow Metab 18:906-910.

de Leeuw FE, and Nichols F, 3rd. (2017). Waxing and waning of white matter hyperintensities. Neurology 89:984-985.

Deprez S, de Ruiter MB, Bogaert S, Peeters R, Belderbos J, De Ruysscher D, Schagen S, Sunaert S, Pullens P, and Achten E. (2018). Multi-center reproducibility of structural, diffusion tensor, and resting state functional magnetic resonance imaging measures. Neuroradiology 60:617-634.

Fan C, Zhao Y, Yu Q, Yin W, Liu H, Lin J, Yang T, Fan M, Gesang L, and Zhang J. (2016). Reversible brain abnormalities in people without signs of mountain sickness during high-altitude exposure. Sci Rep 6:33596.

Fanou EM, Coutinho JM, Shannon P, Kiehl TR, Levi MM, Wilcox ME, Aviv RI, and Mandell DM. (2017). Critical illness-associated cerebral microbleeds. Stroke 48:10851087.

Fayed N, Modrego PJ, and Morales H. (2006). Evidence of brain damage after high-altitude climbing by means of magnetic resonance imaging. Am J Med 119:168.e1-168.e6.

Garcia-Monco JC, Cortina IE, Ferreira E, Martinez A, Ruiz L, Cabrera A, and Beldarrain MG. (2011). Reversible splenial lesion syndrome (RESLES): What's in a name? J Neuroimaging 21:e1-e14.

Garrido E, Castello A, Ventura JL, Capdevila A, and Rodriguez FA. (1993). Cortical atrophy and other brain magnetic resonance imaging (MRI) changes after extremely high-altitude climbs without oxygen. Int J Sports Med 14:232-234.

Garrido E, Segura R, Capdevila A, Aldoma J, Rodriguez FA, Javierra C, and Ventura JL. (1995). New evidence from magnetic resonance imaging of brain changes after climbs at extreme altitude. Eur J Appl Physiol Occup Physiol 70:477-481.

Government of Nepal Ministry of Culture, Tourism \& Civil Aviation, Planning \& Evaluation Division, Research \& Statis- tical Section. (2018). Nepal tourism statistics 2017. Available at: http://tourism.gov.np/publications/1 (accessed September 3, 2019).

Hackett PH, Yarnell PR, Hill R, Reynard K, Heit J, and McCormick J. (1998). High-altitude cerebral edema evaluated with magnetic resonance imaging: Clinical correlation and pathophysiology. JAMA 280:1920-1925.

Hackett PH, Yarnell PR, Weiland DA, and Reynard KB. (2019). Acute and evolving MRI of high-altitude cerebral edema: Microbleeds, edema, and pathophysiology. AJNR Am J Neuroradiol 40:464-469.

Hoffmann A, Kunze R, Helluy X, Milford D, Heiland S, Bendszus M, Pham M, and Marti HH. (2016). High-field MRI reveals a drastic increase of hypoxia-induced microhemorrhages upon tissue reoxygenation in the mouse brain with strong predominance in the olfactory bulb. PLoS One 11: $\mathrm{e} 0148441$.

Hunt JS, Jr., Theilmann RJ, Smith ZM, Scadeng M, and Dubowitz DJ. (2013). Cerebral diffusion and T(2): MRI predictors of acute mountain sickness during sustained highaltitude hypoxia. J Cereb Blood Flow Metab 33:372-380.

Iordanishvili E, Schall M, Loucao R, Zimmermann M, Kotetishvili K, Shah NJ, and Oros-Peusquens AM. (2019). Quantitative MRI of cerebral white matter hyperintensities: A new approach towards understanding the underlying pathology. Neuroimage 202:116077.

Kallenberg K, Bailey DM, Christ S, Mohr A, Roukens R, Menold E, Steiner T, Bartsch P, and Knauth M. (2007). Magnetic resonance imaging evidence of cytotoxic cerebral edema in acute mountain sickness. J Cereb Blood Flow Metab 27:1064-1071.

Kühn S, Noblé H-J, and Knopf H. (2016). Transient splenium lesion-The "reversible splenium lesion syndrome" [in German]. RöFo 188:963-964.

Lawley JS, Alperin N, Bagci AM, Lee SH, Mullins PG, Oliver SJ, and Macdonald JH. (2014). Normobaric hypoxia and symptoms of acute mountain sickness: Elevated brain volume and intracranial hypertension. Ann Neurol 75:890-898.

Lawley JS, Levine BD, Williams MA, Malm J, Eklund A, Polaner DM, Subudhi AW, Hackett PH, and Roach RC. (2016). Cerebral spinal fluid dynamics: effect of hypoxia and implications for high altitude illness. J Appl Physiol 120: 251-262.

Mairer K, Gobel M, Defrancesco M, Wille M, Messner H, Loizides A, Schocke M, and Burtscher M. (2012). MRI evidence: Acute mountain sickness is not associated with cerebral edema formation during simulated high altitude. PLoS One 7:e50334.

McGuire SA, Ryan MC, Sherman PM, Sladky JH, Rowland LM, Wijtenburg SA, Hong LE, and Kochunov PV. (2019). White matter and hypoxic hypobaria in humans. Hum Brain Mapp 40:3165-3173.

Morocz IA, Zientara GP, Gudbjartsson H, Muza S, Lyons T, Rock PB, Kikinis R, and Jolesz FA. (2001). Volumetric quantification of brain swelling after hypobaric hypoxia exposure. Exp Neurol 168:96-104.

Pagani M, Ansjon R, Lind F, Uusijarvi J, Sumen G, Jonsson C, Salmaso D, Jacobsson H, and Larsson SA. (2000). Effects of acute hypobaric hypoxia on regional cerebral blood flow distribution: A single photon emission computed tomography study in humans. Acta Physiol Scand 168:377-383.

Pagani M, Salmaso D, Sidiras GG, Jonsson C, Jacobsson H, Larsson SA, and Lind F. (2011). Impact of acute hypobaric hypoxia on blood flow distribution in brain. Acta Physiol (Oxf) 202:203-209. 
Roach R, Bartsch P, Hackett P, and Oelz O. (1993). The Lake Louise acute mountain sickness scoring system. Hyp Mol Med 272:4.

Rupp T, Jubeau M, Lamalle L, Warnking JM, Millet GY, Wuyam B, Esteve F, Levy P, Krainik A, and Verges S. (2014). Cerebral volumetric changes induced by prolonged hypoxic exposure and whole-body exercise. J Cereb Blood Flow Metab 34:1802-1809.

Sagoo RS, Hutchinson CE, Wright A, Handford C, Parsons H, Sherwood V, Wayte S, Nagaraja S, Ng'Andwe E, Wilson $\mathrm{MH}$, Imray $\mathrm{CH}$; Birmingham Medical Research and Expedition Society. (2017). Magnetic resonance investigation into the mechanisms involved in the development of highaltitude cerebral edema. J Cereb Blood Flow Metab 37:319331.

Sampson JB, Cymerman A, Burse RL, Maher JT, and Rock PB. (1983). Procedures for the measurement of acute mountain sickness. Aviat Space Environ Med 54:1063-1073.

Sanborn MR, Edsell ME, Kim MN, Mesquita R, Putt ME, Imray $\mathrm{C}$, Yow $\mathrm{H}$, Wilson $\mathrm{MH}$, Yodh $\mathrm{AG}$, Grocott $\mathrm{M}$, and Martin DS. (2015). Cerebral hemodynamics at altitude: Effects of hyperventilation and acclimatization on cerebral blood flow and oxygenation. Wilderness Environ Med 26: 133-141.

Schommer K, Bärtsch P, Knauth M, and Kallenberg K. (2012). Teaching Neuroimages: Reversible splenial cytotoxic edema in acute mountain sickness. Neurology 78:932.

Schommer K, Kallenberg K, Lutz K, Bartsch P, and Knauth M. (2013). Hemosiderin deposition in the brain as footprint of high-altitude cerebral edema. Neurology 81:1776-1779.

Schoonman GG, Sandor PS, Nirkko AC, Lange T, Jaermann T, Dydak U, Kremer C, Ferrari MD, Boesiger P, and Baumgartner RW. (2008). Hypoxia-induced acute mountain sickness is associated with intracellular cerebral edema: A $3 \mathrm{~T}$ magnetic resonance imaging study. J Cereb Blood Flow Metab 28:198-206.

Swenson ER, and Bärtsch P. (2014). High Altitude Human Adaption to Hypoxia. Springer Science+Business Media New York, New York.

Verges S, Rupp T, Villien M, Lamalle L, Tropres I, Poquet C, Warnking JM, Esteve F, Bouzat P, and Krainik A. (2016). Multiparametric magnetic resonance investigation of brain adaptations to 6 days at $4350 \mathrm{~m}$. Front Physiol 7:393.

Villien M, Bouzat P, Rupp T, Robach P, Lamalle L, Tropres I, Esteve F, Krainik A, Levy P, Warnking JM, and Verges S.
(2013). Changes in cerebral blood flow and vasoreactivity to $\mathrm{CO}_{2}$ measured by arterial spin labeling after 6 days at $4350 \mathrm{~m}$. Neuroimage 72:272-279.

Wardlaw JM, Chappell FM, Valdes Hernandez MDC, Makin SDJ, Staals J, Shuler K, Thrippleton MJ, Armitage PA, Munoz-Maniega S, Heye AK, Sakka E, and Dennis MS. (2017). White matter hyperintensity reduction and outcomes after minor stroke. Neurology 89:1003-1010.

Wardlaw JM, Valdes Hernandez MC, and Munoz-Maniega S. (2015). What are white matter hyperintensities made of? Relevance to vascular cognitive impairment. J Am Heart Assoc 4:001140.

Wilson MH, Imray CH, and Hargens AR. (2011). The headache of high altitude and microgravity-Similarities with clinical syndromes of cerebral venous hypertension. High Alt Med Biol 12:379-386.

Yamauchi H, Pagani M, Fukuyama H, Ouchi Y, Nagahama Y, Matsuzaki S, Kimura J, Yonekura Y, and Konishi J. (1995). Progression of atrophy of the corpus callosum with deterioration of cerebral cortical oxygen metabolism after carotid artery occlusion: A follow up study with MRI and PET. J Neurol Neurosurg Psychiatry 59:420-426.

Zhang H, Lin J, Sun Y, Huang Y, Ye H, Wang X, Yang T, Jiang $\mathrm{X}$, and Zhang J. (2012). Compromised white matter microstructural integrity after mountain climbing: Evidence from diffusion tensor imaging. High Alt Med Biol 13:118-125.

Zhang J, Zhang H, Li J, Chen J, Han Q, Lin J, Yang T, and Fan M. (2013). Adaptive modulation of adult brain gray and white matter to high altitude: Structural MRI studies. PLoS One 8: e68621.

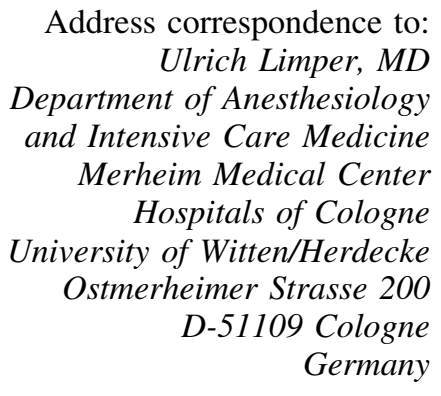

E-mail: ulrich.limper@dlr.de 